Rev Biomed 2006; 17:81-84.

\title{
El cáncer cérvico-uterino, su impacto en México y el porqué no funciona el programa nacional de detección oportuna.
}

\section{Carta al Editor}

\begin{abstract}
Ana C. Hidalgo-Martínez.
Centro de Investigación sobre Enfermedades Infecciosas. Instituto Nacional de Salud Pública, Cuernavaca, Morelos, México.
\end{abstract}

La infección genital con el virus del papiloma humano (VPH) es la enfermedad de transmisión sexual viral más frecuente a nivel mundial (1). Asimismo, es el factor de riesgo más importante para desarrollar lesiones preneoplásicas y neoplásicas del cuello uterino (2). No obstante, menos de 5\% de las mujeres infectadas con el VPH desarrollarán cáncer cérvicouterino $(\mathrm{CaCu})(3)$.

$\mathrm{El} \mathrm{CaCu}$ es un problema de salud pública; es la enfermedad neoplásica más frecuente y mortal en la población femenina. Cada año 500,000 casos nuevos son diagnosticados en todo el mundo (4). En el año 2001 , el $11.7 \%$ de todas las neoplasias en las mujeres correspondieron a $\mathrm{CaCu}$, y se reportaron 369,500 casos nuevos en países en vías de desarrollo, a diferencia de los países desarrollados en los cuales 96,100 casos fueron diagnosticados en el mismo año (5).

La población femenina originaria de Latinoamérica es considerada como de alto riesgo para desarrollar $\mathrm{CaCu}$. Cada año se reportan 68,000 casos nuevos. Estudios comparativos de las tasas de mortalidad por esta patología, señalan que tasas más altas corresponden a Chile y México y las más bajas a Cuba, Puerto Rico y Argentina (6).

En México el $\mathrm{CaCu}$ es la primera causa de muerte por neoplasias en mujeres mayores de 25 años (7). El Sistema Nacional de Salud Mexicano brinda atención médica aproximadamente a 9,000 casos de $\mathrm{CaCu}$ invasor y se registran 4,000 muertes anualmente (2). En el año 2001, se reportaron 4,051 muertes en mujeres por $\mathrm{CaCu}$, con una tasa de mortalidad de 8.8 por cada 100,000 mujeres. Para el año 2002 se registraron 4,323 casos con una tasa de 8.6 por 100,000 mujeres (8).

Sin embargo, este tipo de cáncer es absolutamente prevenible y su tratamiento es relativamente fácil, cuando el diagnóstico es oportuno. Sabemos que es de etiología infecciosa, pero desde la perspectiva de la salud pública, estamos conscientes de que los programas de control no han funcionado como se esperaba. La experiencia de países desarrollados ha permitido demostrar que la mejor opción para disminuir la mortalidad por $\mathrm{CaCu}$ es la detección y el tratamiento oportuno de lesiones precursoras y lesiones malignas por medio de programas de detección oportuna del $\mathrm{CaCu}(9)$.

Aunque existe un programa nacional de

Solicitud de sobretiros:Ana C. Hidalgo-Martínez. Calle Invierno No. 5-B, Colonia Universidad, C.P. 62217, Cuernavaca, Morelos, México. Tel 01(777) 1029141, Correo electrónico: ahidalgo@espm.insp.mx 


\section{AC Hidalgo-Martínez.}

Detección Oportuna del Cáncer (DOC), mediante la prueba de Papanicolaou (Pap), desde 1974 en México, el $\mathrm{CaCu}$ sigue siendo una de las principales causas de muerte para las mujeres mexicanas (10). En nuestro país ha sido difícil establecer y mantener un programa de tamizaje efectivo, tal como los programas de los países desarrollados, que sí han logrado disminuir sus tasas de mortalidad por $\mathrm{CaCu}$ (11-13).

El examen de Pap no es un examen de diagnóstico. Es una prueba de tamizaje que detecta a las mujeres que pueden tener lesiones en el cuello del útero de las que no las tienen, las cuales son la mayoría. Los resultados del examen no siempre son "exactos". En ocaciones la citología es positiva, pero finalmente la mujer no tiene lesiones precursoras o lesiones malignas; y en otras, la citología es negativa y resulta que si había cambios anatómicos de malignidad, que no fueron detectados.

Desgraciadamente, esta última situación, que es bastante grave, es también común. Ahora se sabe que solamente en la mitad de las mujeres que tienen cambios morfológicos, la citología es capaz de detectar estos cambios. Es decir, estamos utilizando un examen que no es del todo el más adecuado o el personal que observa al microscopio las laminillas no está capacitado para poder establecer con precisión el resultado correcto. O la carga de trabajo es tanta, que puede cometer errores involuntarios, ya que se pasan horas frente al microscopio al día.

En en vez de ser una herramienta para la DOC, el Pap frecuentemente detecta casos de $\mathrm{CaCu}$ ya muy avanzados. Entre el 20 y $60 \%$ de las muertes por $\mathrm{CaCu}$ se podrían prevenir mediante el uso efectivo y temprano de un programa de DOC (14). Se estima que el actual programa de DOC previene menos de $13 \%$ de los casos de $\mathrm{CaCu}$ potencialmente prevenibles en México (15).

El hecho de que gran parte de estos casos de $\mathrm{CaCu}$ se desarrollen en mujeres de escasos recursos o que tienen un acceso limitado a los servicios de salud, muchas de ellas no se hacen la prueba de Pap, que es la más conocida para detectar el $\mathrm{CaCu}$. Las razones son variadas, entre las que destacan el hecho de que no han sido suficientemente informadas acerca de los beneficios para su salud, lo cual es un indicador que la información del programa de la DOC no ha permeado lo suficiente en las mujeres y sus parejas. Otra variable puede ser, que no tienen acceso a los centros de salud que lo proporcionan gratuitamente $o$ que han tenido experiencias negativas con el médico u otros profesionales de salud que los atendió. También hay que considerar la educación y la formación familiar que han recibido, la cual, no les permite sopesar la importancia de esta prueba. Así, la mayoría de las mujeres, da absoluta prioridad a la salud de su familia por sobre la propia, tiene miedo de la opinión o reacción de su pareja, quien puede prohibirle que vaya al ginecólogo. Por último, no entendemos el pudor de gentes del área rural, condición prioritaria para poder hacerles llegar el beneficio de la DOC que puede salvarles la vida.

Por otra parte, en América Latina esta prueba está estrechamente ligada a los programas de planificación familiar. Por ende, el tamizaje se hace a las mujeres menores de 30 años de edad. Pero, puesto que el grupo de mujeres que corre el riesgo más alto de tener $\mathrm{CaCu}$ es de 25 a 60 años, los programas de prevención y detección no son tan eficaces como deberían serlo en mujeres de más de 30 años.

El acceso a los servicios médicos, listas de espera y la recuperación de los costos del tratamiento son graves problemas en América Latina. Si bien los exámenes de Pap son gratuitos en la mayoría de los casos, el diagnóstico confirmatorio y tratamiento posteriores pueden no serlo.

A menudo las pacientes carecen de seguro médico, o los centros médicos para tratamientos de nivel secundario o terciario están alejados; sin mencionar el hecho de que la mujer debe realizar múltiples visitas al médico para recibir el diagnóstico, los resultados y la hospitalización en caso de requerirla. Vale la pena acotar en este parrafo, que es posible que el Seguro Popular que implemento el Gobierno de México a través de la Secretaría de Salud, pueda cubrir no sólo el Pap, sino todo lo que implica el diagnóstico confirmatorio, el tratamiento y seguimento de la paciente. Pero lo más importante aún es

\section{Revista Biomédica}




\section{Detección del cáncer cervico-uterino.}

reestructurar el programa de DOC, ya que si se continua con el mismo programa, a pesar del Seguro Popular y la creación de las clínicas de displasias y la utilización de colposcopía en todo el país, el $\mathrm{CaCu}$ seguirá igual, ya que el problema no es la infraestructura en sí, sino que las mujeres no acuden por falta de sensibilización médica, moral y ética a ser examinadas cada año.

Las diferencias socioeconómicas y algunos enfoques mal especificados de los servicios médicos han dado pie para que muchas mujeres perciban los exámenes de diagnóstico más bien como una fase de prevención. Por lo tanto, muchas mujeres piensan que basta con hacerse el Pap una vez y ya están "curadas" o no van a sufrir de $\mathrm{CaCu}$ nunca. Cerca de un tercio de ellas ni siquiera vuelve a buscar los resultados y aquellas que lo hacen, tienen grandes dificultades para comprender las explicaciones, si es que hay explicaciones. En caso de ser positiva la prueba del Pap, se les dice que necesitan otra consulta, que es necesario realizar más exámenes, porque es posible que tengan un cáncer. Esto provoca desesperación entre las mujeres, especialmente entre las de menores recursos y educación, porque les resulta difícil comprender y acudir a otras consultas, por el tiempo y el costo que esto tiene para su familia.

La etiología y nosología de la enfermedad tiene diferentes significados para el equipo médico y para las mujeres. Para estas últimas, las causas de la enfermedad tienden a vincularlo con prácticas que son valoradas de manera negativa en su cultura, tienden a mencionar con frecuencia que se debe a que las mujeres tienen diferentes parejas sexuales y no mencionan en el mismo nivel de preocupación las relaciones de sus parejas con otras mujeres y que son ellos los portadores del VPH. Aparentemente no hay una vinculación clara entre las prácticas sexuales de los hombres con el cáncer que ellas consideran como propio de las mujeres. El principio de no maleficencia considera la protección del paciente en forma adecuada, a través de medidas efectivas de prevención, así como la protección de terceros en riesgo, por lo cual la DOC se debería de hacer llegar de igual manera a los hombres al igual que las mujeres, ya que se sabe que el principal cofactor del $\mathrm{CaCu}$ es un virus, considerado como una infección viral y que no sólo es por una parte, sino que juega un papel importante la pareja.

La "prueba del cáncer" es una experiencia que las mujeres viven solas, pero que en su mayoría desean vivirla así. Mostrar la parte más intima de su cuerpo a otros, aún considerando que sea su pareja o familiar cercano, no es una experiencia que se viva sin sentimientos de vergüenza fuera de los contextos permitidos a plena luz del día. Estos sentimientos se relacionan con los procesos de socialización que van configurando la formación de las identidades de género. El cuerpo censurado y controlado en la mayoría de los espacios de la vida cotidiana, es una vivencia que repercute en las dificultades para acudir a la prueba del Pap y posiblemente en las campañas de DOC.

Por la etiología y nosología del cáncer para las mujeres, el haber tenido experiencias directas o indirectas con la enfermedad en cualquier lugar del cuerpo, marca en gran medida su práctica de acudir a la DOC. La misma puede ser en dos sentidos, agudizando el miedo al resultado y con ello las dificultades para acudir a la detección o bien, en el otro sentido, motivando la práctica de manera frecuente. Esto en gran medida dependerá del nivel de educación de cada mujer y comprender esta situación, es un aspecto que se debe de analizar en las campañas de DOC.

Las palabras y el tono que utiliza el personal de salud para referirse al procedimiento o a sus observaciones, forman parte de las experiencias vividas de las mujeres que ellas consideran para regresar o no al mismo lugar y aún para considerar con mayor o menor dificultad el volver a realizar la DOC de manera oportuna. La confianza es un ingrediente esencial en las interacciones entre médicos y pacientes. Una confianza basada no en la autoridad y en seguir órdenes del médico, sino en esperanza, confianza digna de fe, de que el médico ayudará al paciente a tomar sus propias decisiones.

Por lo tanto el programa de detección oportuna del cáncer debería:

Vol.15/No.4/Octubre-Diciembre, 2004 


\section{AC Hidalgo-Martínez.}

1) Tener entrenamiento permanente de educación y sensibilización para prestadores de servicios de salud, encaminado a fomentar la utilización del programa y mejorar el compromiso al mismo, con el fin de incrementar la cobertura, por lo que es necesario que el personal de salud esté formado en todos los campos de la atención, iniciando por el trato amable y respetuoso. Deberá ser conocedor de los valores generales de la moralidad de la sociedad que están a su cargo.

2) Incluir en el programa de DOC otras pruebas, como son la toma de muestras para la prueba de VPHclínico (ensayo de captura de híbridos), que tiene mayor sensibilidad y las mujeres que no están infectadas con VPH podrían ser tamizadas en intervalos más largos. La colposcopía debería ser usada de manera rutinaria en la DOC, no sólo en la consulta privada, sino a todos los niveles.

3) Establecer mecanismos de capacitación y acreditación del personal encargado de la toma del espécimen para la prueba, así como de la interpretación diagnóstica del mismo, aunado a un sistema de control de calidad de estos procedimientos.

4) Crear un sistema automatizado de vigilancia epidemiológica para el seguimiento de la población en riesgo, que permita el monitoreo continuo y la evaluación sistemática del programa.

Palabras clave: cáncer cervico-uterino, detección oportuna de cáncer, Papanicolaou, virus de papiloma humano.

\section{REFERENCIAS.}

1.- Bosch FX, Manos MM, Munoz N, Sherman M, Jansen AM, Peto J, et. al. Prevalence of human papillomavirus in cervical cancer: a worldwide perspective. International biological study on cervical cancer (IBSCC) Study Group. J Natl Cancer Inst 1995; 87:796-802.

2.- Walboomers JM, Jacobs MV, Manos MM, Bosch FX, Kummer JA, Shah KV, et al. Human papillomavirus is a necessary cause of invasive cervical cancer worldwide. J Pathol 1999; 189:12-9.

3.-Ho GY, Burk RD, Klein S, Kadish AS, Chang CJ, Palan P, et al. Persistent genital human papillomavirus infection as a risk factor for persistent cervical dysplasia. J Natl Cancer Inst 1995;87:1345-7.

4.- Parkin DM, Pisani P, Ferlay J. Estimates of the worldwide incidence of eighteen major cancers in 1985. Int J Cancer 1993; 54:594-606.

5.- Taller sobre vinculación de la investigación epidemiológica en programas de prevención y control de cáncer. Salud Pública de México 1995; 4:375-80.

6.- Robles SC, White F, Peruga A. Trends in cervical cancer mortality in the Americas. Bull Pan Am Health Organ 1996; 30:290-301.

7.- Manual de normas y procedimientos. Prevención de los cánceres cervicouterino y mamario. Estados Unidos Mexicanos. Secretaria de Salud. Dirección General de Medicina Preventiva, 1993.

8.- Estadísticas de mortalidad en México: Muertes registradas en el 2001. Salúd Pública de México 2002; 44:56581.

9.- The World Health Report 1995. Bridging the gaps. World Health Organization, Geneva. http://www.who.int/whr/1995/ en/whr95_en.pdf.

10.- Lazcano-Ponce EC, Rascon-Pacheco RA, LozanoAscenci R, Velasco-Mondragón HE. Mortality from cervical carcinoma in Mexico: Impact of screening, 1980-1990. Salud Publica Mex 1997; 39:266-73.

11.- Ordoñez BR. Avances en la prevención del cáncer cervicouterino en el Instituto Mexicano del Seguro Social. Salud Publica Mex 1971; 13: 327-9.

12.- Manual para registro histopatológico de neoplasias malignas. Estados Unidos Mexicanos. Secretaria de Salud. Dirección General de Epidemiología, 1996.

13.- Hernández-Ávila M, Lazcano-Ponce EC, Alonso de Ruiz P, López-Carrillo L, Rojas-Martínez R. Evaluación del programa de detección oportuna del cáncer del cuello uterino enla ciudad de México: un estudio epidemiológico de casos y controles con base poblacional. Gac Med Mex 1994; 130:201-9.

14.- Lazcano-Ponce EC, Nájera-Aguilar P, Alonso de Ruiz P, Buiatti E, Hernández-Ávila M. Programa de detección oportuna de cáncer cervical en México. Diagnóstico situacional. Cancerología 1996; 42:123-40.

\section{Revista Biomédica}

